\title{
What Obstetrician-Gynecologists Think of Preconception Care
}

\author{
Maria A. Morgan · Debra Hawks · Stanley Zinberg • \\ Jay Schulkin
}

Published online: 7 June 2006

(C) Springer Science+Business Media, Inc. 2006

\begin{abstract}
Objectives: To describe obstetrician-gynecologists' opinions of preconception care (PCC) and ascertain patient uptake for this service. Methods: A questionnaire was mailed to 1105 ACOG members in August 2004. Results: There was a $60 \%$ response rate. Most physicians think PCC is important $(87 \%)$ and almost always recommend it to women planning a pregnancy (94\%); 54\% do so with women who are sexually active. Around a third (34\%) thought their patients usually do not plan their pregnancies and $49 \%$ said very few pregnant patients came in for PCC. Of those who obtain PCC, they were believed to do so more likely to assure a healthy pregnancy $(83 \%)$ than because of an elevated risk for birth defects (20\%). Of 11 issues presented, cigarette smoking and folic acid supplementation were rated the most important for PCC counseling; exercise and environmental concerns were the least important. Conclusions: Physicians are willing to provide PCC but few patients are accessing such services.
\end{abstract}

Keywords Preconception care · Survey ·

Obstetrician-gynecologist $\cdot$ Routine care $\cdot$ Folic acid

\section{Introduction}

When poor pregnancy outcomes occur, they frequently have been set in motion long before the first prenatal visit. Fortunately, many of the factors contributing to less than ideal birth outcomes can be managed and brought under control

M. A. Morgan ( $\bowtie) \cdot$ D. Hawks · S. Zinberg · J. Schulkin Research Department, American College of Obstetricians and Gynecologists,

409, 12th Street, S.W., Washington, DC 20024, USA.

e-mail:mmorgan@acog.org prior to conception. Preconception care (PCC) consists of the identification of those conditions that could affect a future pregnancy or fetus and that may be amenable to intervention [1]. ACOG recommends that all health encounters during a woman's reproductive years, particularly those that are a part of PCC, should include counseling on appropriate medical care and behavior to optimize pregnancy outcomes [1]. Through PCC, physicians can advise patients on numerous behavioral and lifestyle changes, ranging from exercise and weight control to the use of prescribed medications and illicit drugs. They may also address carrier screening for heritable genetic disorders and the options available for helping to avoid an affected birth. For example, it is well known that women with a chronic disease such as diabetes have an increased risk of congenital abnormalities in their offspring, and they are known to have improved birth outcomes when they plan their pregnancies and utilize PCC [2]. Another prime example of the success of PCC efforts is the reduction in the number of pregnancies affected by neural-tube defects following national efforts to increase consumption and supplementation of folic acid by women of childbearing potential [3] through education and food fortification. Despite such improvements, fewer than 50\% of women are aware of the benefits of taking folic acid supplements around the time of conception [4], the majority of women with diabetes do not plan their pregnancies [5], many women continue to drink alcohol regularly during pregnancy [6] and almost 50\% of pregnancies in the U.S. are unplanned [7].

PCC has had its successes, but clearly more work needs to be done. The importance of PCC in promoting maternal and fetal health has long been recognized, and national recommendations and guidelines for PCC are being developed [8]. One aspect of successful development of PCC guidelines involves knowledge of obstetrician-gynecologists' current practices and opinions regarding PCC and what they 
perceive to be barriers to successful implementation of PCC. The purpose of this study was to assess the practices and opinions of obstetrician-gynecologists regarding PCC, and how frequently they perceive their patients to avail themselves of such services.

\section{Materials and methods}

Questionnaires were mailed in August 2004 to 1105 American College of Obstetricians and Gynecologists (ACOG) Fellows and Junior Fellows in Practice. Of these subjects, 605 were members of the Collaborative Ambulatory Research Network (CARN), and 500 were a computer-generated random sample of ACOG members who had not received a survey from ACOG during the previous two years (NonCARN). Members of CARN are practicing obstetriciangynecologists who have volunteered to participate in survey studies on a regular basis. CARN was established to facilitate assessment of clinical practice patterns and aid the development of educational materials. Two reminder mailings were sent to non-respondents and questionnaires returned by December 31, 2004 were included in the survey. This protocol has typically resulted in a total sample size of $>450$, which is sufficient to detect differences between groups of $<0.5$ standard deviation with power of $80 \%$ and significance at the 0.05 level [9].

The survey recorded demographic details of physicians and their patient population, and assessed practices and opinions regarding PCC. A questionnaire concerning screening for aneuploidy was included in the same mailing; both questionnaires shared a demographics section containing 9 questions on one page. The PCC questionnaire contained 11 questions, some multi-part, on two pages. Both questionnaires were brief and resulted in a final document of 3 double-sided pages, a standard length for our full-length questionnaires. Seven of the 11 questions on PCC involved rating the frequency of an activity (e.g., always, usually, occasionally, never) or degree of agreement with a statement, and three questions were of a multiple-choice format. The questionnaires were developed in consultation with medical specialists and were pilot tested on a sample of practicing obstetrician-gynecologists prior to final distribution.

The data were analyzed using a personal computer-based software package (SPSS ${ }^{\circledR}$ 12.0, SPSS Inc., Chicago, IL). Descriptive statistics were computed for the measures used in the analyses, which are reported as mean \pm SEM. Student's $t$ test was used to compare group means of continuous variables. Factor Analysis was conducted on two sets of rating variables (Agreement with 6 statements regarding PCC, and Importance of counseling on 11 issues during PCC and during routine care), and factors with eigenvalues greater than one were treated with a varimax rotation. Differences in ratings on the Importance scales were examined with multivariate analysis of variance. Differences on categorical measures were assessed using $\chi^{2}$. Group differences on ordinal measures were assessed using the Mann-Whitney U test or Kruskal Wallis $\chi^{2}$. Related-sample differences on ordinal measures used the Wilcoxon signed ranks test. Correlations including an ordinal measure used the Spearman's rho coefficient. All analyses were tested for significance using an alpha of 0.05 .

\section{Results}

\section{Demographics}

A total of 670 questionnaires were returned. Data from 18 respondents were judged invalid (physician retired, returned to sender), resulting in a valid response rate of $60 \%(652 / 1087)$, 432 from CARN members ( $72.1 \%$ response rate) and 220 from Non-CARN (45.1\% response rate). Physicians responded from every state of the United States except Maine, as well as the District of Columbia, Puerto Rico, Canada, and overseas military installations. The respondents' mean age $(47.32 \pm 0.39)$ and the proportion of males to females $($ males $=55 \%)$ closely matched those of the larger population to whom the survey was sent $(46.99 \pm 0.32$, males $=$ $55 \%)$ and of ACOG Fellows and Junior Fellows in Practice as a whole $(47.64$, males $=58 \%)$.

The remaining analyses are limited to the $88.8 \%$ (579) of respondents who indicated that their primary medical specialty was gynecology or general obstetrics and gynecology. This selection was made to best assess the practices of non-subspecialist obstetricians and gynecologists, rather than those in maternal fetal medicine, reproductive endocrinology, or other subspecialties, to whom patients may be referred after pregnancy has already been attempted. Of these physicians, $83.9 \%$ (486) practice obstetrics (ObGyn), and $16.1 \%$ (93) do not (GynOnly); the term 'physicians' is used when referring to both ObGyns and GynOnlys. See Table 1 for physician demographics. CARN and Non-CARN differed on only one non-demographic item (CARN were more likely than Non-CARN to discuss carrier screening with all patients who present for PCC. See results.); thus data were collapsed across these two groups.

\section{Defining preconception care}

Physicians were asked whether they would define PCC as specialized or routine care. The great majority $(86.7 \%)$ defined it "As specialized pre-pregnancy care that focuses on issues not typically addressed during a routine exam which are specific to ensuring an optimal pregnancy outcome." Far fewer (13.3\%) defined it as "The same as routine 
Table 1 Physician demographics

\begin{tabular}{ll}
\hline & $\begin{array}{l}\text { Total } \\
(n=579)\end{array}$ \\
\hline Gender $(\%)$ & \\
Males & 53.9 \\
Females & 46.1 \\
CARN & 67.5 \\
Non-CARN & 32.5 \\
Age in years, mean (SEM)* & $47.12(0.42)$ \\
Males & $50.88(0.54)$ \\
Females & $42.76(0.54)$ \\
Years in practice, mean (SEM) & $15.22(0.41)$ \\
Deliveries in 2003, mean (SEM) & $129.65(3.13)$ \\
Practice location (\%) & \\
Urban, inner city & 10.7 \\
Urban, non-inner city & 29.3 \\
Suburban & 32.1 \\
Mid-sized town & 19.4 \\
Rural & 6.9 \\
Other & 1.6 \\
Practice type (\%) & \\
Ob/Gyn partnership/gp & 51.4 \\
Solo practice & 22.5 \\
Multi-specialty & 10.4 \\
University full-time faculty and practice & 8.5 \\
Other & 7.2 \\
Patient ethnicity-mean (SEM) \% of patients & \\
Non-Hispanic white & $62.29(1.10)$ \\
African-American & $16.78(0.79)$ \\
Hispanic & $13.35(0.78)$ \\
Asian/Pacific Islander & $3.83(0.29)$ \\
Native American & $1.29(0.23)$ \\
\hline & \\
\hline & \\
&
\end{tabular}

${ }^{*} P<0.001$ : Males older than females; CARN older than non-CARN.

well-woman care that occurs during the reproductive years, prior to a pregnancy." Those who defined PCC as routine tended to be in practice longer than those defining it as specialized $(17.55 \pm 1.16 \mathrm{yrs}$ versus $14.84 \pm 0.45$ yrs. $t(555)=2.22 ; P<0.05)$, independent of gender.

\section{Recommending PCC}

The vast majority of physicians (97.3\%) indicated that they provide PCC for their patients. Physicians were asked how frequently they recommend PCC to different groups of women of childbearing age (see Table 2). Three quarters (75\%) always recommend PCC to patients planning a pregnancy, and 9 in 10 (89.9\%) always recommend it to diabetic patients planning a pregnancy. Physicians who defined PCC as routine were more likely than those who defined it as specialized to recommend PCC to women who are sexually active ('always' or 'usually': routine $=63.4 \%$, specialized $=$ $52.1 \%$. M-W $U=13989.5 ; P<0.05)$ and to women who are using birth control ('always' or 'usually': routine $=48.6 \%$, specialized $=32.8 \%$. M-W U $=13002 ; P<0.01)$.
Opinions about PCC

Physicians were asked their level of agreement with several statements regarding PCC (see Table 3). The majority agreed (4-5 on 5 point scale) that PCC is an important issue $(87.3 \%)$ and that it has a positive effect on pregnancy outcomes $(83.5 \%)$, though only $20.7 \%$ agreed that it is a high priority in their workload. Three quarters $(76.8 \%)$ thought they have appropriate training to provide PCC. Half agreed that there is not enough time to provide preconception care visits to all women of childbearing age (51.4\%), and that time devoted to PCC is not reimbursed (49.8\%). Based on factor analysis, the first three items were combined to produce a single mean score for the 'positive' aspects of PCC (Factor I), and the second set of three items for the 'negative' aspects of PCC (Factor II). There were significant positive correlations between agreement on Factor I and frequency of recommending PCC in all groups of women (all $P$ 's $<0.001$ ); there were significant negative correlations between agreement on Factor II and frequency of recommending PCC in all groups of women (all $P$ 's $<0.01$ ). In other words, the more strongly they agreed that PCC was important/positive/high priority, the more frequently they recommended PCC. Looking at the individual components of the two factors, lack of reimbursement for PCC was the only item that did not correlate with frequency of recommending PCC in any group of women.

\section{Counseling in PCC}

Almost a third $(31.9 \%)$ of physicians said they discuss screening for aneuploidy with All patients who present for preconception counseling. Of those not selecting All, $80.7 \%$ said they did so with patients at risk for aneuploidy. Almost one-in-ten (9.8\%) said they did not discuss aneuploidy. The majority (54.4\%) of physicians said they discuss carrier screening for heritable genetic disorders (e.g., CF, sickle cell anemia) with All patients who present for preconception counseling, and, of those not selecting All, $84.8 \%$ said they did so with patients who have a family history of heritable genetic disorders or other risk factors. Only $4.3 \%$ said they did not discuss carrier screening.

Physicians were presented with 11 health-related issues (e.g., exercise, nutrition, over the counter drug use) and were asked to rate how important counseling was for each issue during a routine office exam and during preconception care (see Table 4). (Note: Physicians were not asked to rank order the issues in terms of relative importance, nor were they asked to compare the issues in terms of routine or PCC; such calculations were made based on analyzing the mean ratings for each issue.) Physicians rated all issues as more important in PCC than in routine care, although the difference for Exercise was marginal (Wilcoxon signed ranks test. 
Table 2 Percent of physicians indicating how frequently they recommend PCC to different groups of women

\begin{tabular}{llccc}
\hline $\begin{array}{l}\text { How frequently do you recommend preconception } \\
\text { care to the following women of childbearing age? }\end{array}$ & Always & Usually & Occasionally & Never \\
\hline Diabetic women planning a pregnancy & 89.9 & 7.9 & 1.7 & 0.5 \\
Women who are planning a pregnancy & 75.0 & 19.2 & 5.0 & 0.8 \\
Obese women planning a pregnancy & 61.5 & 27.0 & 9.5 & 2.0 \\
Women indicating they want children in the future & 38.5 & 43.5 & 16.0 & 2.0 \\
Women who are sexually active & 19.1 & 34.9 & 38.6 & 7.4 \\
Women who are using birth control & 11.5 & 24.1 & 45.9 & 18.5 \\
\hline
\end{tabular}

Exercise: $P=0.052$. All other $P$ 's $<0.001)$. All issues were rated as very important for $\mathrm{PCC}$, with cigarette smoking and folic acid supplementation rated the most highly important issues in PCC. Cigarette smoking and illegal drug use were rated the most important issues for counseling in routine care. Whereas folic acid supplementation ranked second in importance for PCC, it ranked tenth for routine care.

The 11 issues were grouped into 4 factors, for both routine care and PCC (see item labels, Table 4). How physicians defined PCC had a significant effect on importance ratings overall, controlling for years in practice $[F(8,539)=3.437$; $P<0.005]$. However, the only individual factor on which ratings were distinctly different was folic acid supplementation during routine care: physicians who defined PCC as routine were more likely than those defining it as specialized to rate it as very important ( $49.3 \%$ vs $34.8 \%$ ) during routine care $[t(556)=2.53 ; P<0.02]$.

Gender, controlling for years in practice, also had an overall effect on ratings $[F(8,541)=4.066 ; P<0.001]$ : females tended to rate most items as more highly important than did males.

Patient use of PCC

Of the physicians who provide PCC, a third (32.3\%) said that patients 'rarely' present for PCC, almost half (48.5\%) said they 'sometimes' do, and $17.3 \%$ said patients 'frequently' present for PCC. Over a third (34.7\%) agreed that their patients usually do not plan their pregnancies. Almost half (49.1\%) of ObGyns said 'few' or 'none' of their pregnant patients came in for PCC prior to the pregnancy, and almost two thirds $(63.1 \%)$ said 'most' or 'all' of their pregnant patients initially made contact with them once they were already pregnant. See Table 5. The frequency with which patients reportedly present for PCC was positively correlated with physician agreement that their patients plan their pregnancies $(P<0.001)$, as well as with how frequently physicians recommend PCC to the 6 different patient groups listed in Table 2 (all $P$ 's $<0.001$, except 'Diabetic,' $P=0.01$ ).

Physicians were asked to indicate how many of the patients who presented for PCC did so for each of three reasons (7 point scale: None Few Some Half Many Most All). Over four-fifths $(82.7 \%)$ of physicians indicated that more than half of the patients do so to ensure a healthy pregnancy (mean $5.63 \pm 0.062$ on $7 \mathrm{pt}$ scale). In contrast, $41.9 \%$ said more than half do so because of difficulties conceiving (mean $4.05 \pm .058$ ), and $20.0 \%$ said more than half do so because of an elevated risk of a birth defect or developmental disorder (mean $3.13 \pm 0.064$ ).

\section{Discussion}

The purpose of this study was to examine obstetriciangynecologists' opinions and beliefs about PCC, and how

Table 3 Percent of physicians indicating how strongly they agree with several statement regarding PCC

\begin{tabular}{|c|c|c|c|c|c|c|}
\hline Six statements regarding opinions about PCC & $\begin{array}{l}\text { Strongl } \\
\text { agree } \\
5\end{array}$ & 4 & 3 & 2 & $\begin{array}{l}\text { Strongly } \\
\text { disagree } \\
1\end{array}$ & $\begin{array}{l}\text { Mean on } 5 \text { pt } \\
\text { scale (SEM) }\end{array}$ \\
\hline \multicolumn{7}{|l|}{ Factor I: positive aspects } \\
\hline Preconception care is an important issue & 47.4 & 39.9 & 10.5 & 1.3 & 0.9 & $4.32(0.034)$ \\
\hline Preconception care has a positive effect on pregnancy outcomes & 44.7 & 38.8 & 14.3 & 1.1 & 1.1 & $4.25(0.035)$ \\
\hline Preconception care is a high priority in my workload & 6.8 & 13.9 & 42.3 & 27.9 & 9.1 & $2.82(0.043)$ \\
\hline \multicolumn{7}{|l|}{ Factor II: negative aspects } \\
\hline Time devoted to preconception care is not reimbursed & 24.7 & 25.1 & 30.8 & 14.0 & 5.5 & $3.50(0.051)$ \\
\hline $\begin{array}{l}\text { There is not enough time to provide preconception care visits to } \\
\text { all women of childbearing age }\end{array}$ & 22.6 & 28.8 & 19.7 & 18.9 & 10.0 & $3.35(0.056)$ \\
\hline I do not have appropriate training to provide preconception care & 3.0 & 5.2 & 14.7 & 35.7 & 41.4 & $1.92(0.044)$ \\
\hline
\end{tabular}


Table 4 Percent of physicians indicating how important they think counseling is on several issues for patients who (a) come in for a routine office exam, and (b) those who are planning a pregnancy

\begin{tabular}{|c|c|c|c|c|c|}
\hline & $\begin{array}{l}\text { (Rank of mean } \\
\text { Importance } \\
\text { within a or b) }\end{array}$ & $\begin{array}{l}\text { Very important } \\
{[6-7]}\end{array}$ & Neutral [3-5] & $\begin{array}{l}\text { Not important } \\
{[1-2]}\end{array}$ & $\begin{array}{l}\text { Mean (SEM) } \\
\text { on } 7 \text { pt scale }\end{array}$ \\
\hline \multicolumn{6}{|l|}{ Factor I } \\
\hline \multicolumn{6}{|l|}{ Folic acid supplements: } \\
\hline (a) routine exam & (10) & 36.8 & 52.5 & 10.7 & $4.88(0.07)$ \\
\hline (b) preconception care & (2) & 96.4 & 3.4 & .2 & $6.83(0.03)$ \\
\hline \multicolumn{6}{|l|}{ Factor II } \\
\hline \multicolumn{6}{|l|}{ Cigarette smoking: } \\
\hline (a) routine exam & (1) & 89.2 & 10.6 & 0.2 & $6.57(0.03)$ \\
\hline (b) preconception care & (1) & 98.4 & 1.6 & 0.0 & $6.86(0.02)$ \\
\hline \multicolumn{6}{|l|}{ Illegal drug use: } \\
\hline (a) routine exam & (2) & 83.2 & 15.2 & 1.6 & $6.40(0.05)$ \\
\hline (b) preconception care & (3) & 94.5 & 5.1 & 0.4 & $6.74(0.03)$ \\
\hline \multicolumn{6}{|l|}{ Alcohol consumption: } \\
\hline (a) routine exam & (7) & 49.1 & 46.5 & 4.4 & $5.40(0.06)$ \\
\hline (b) preconception care & (4) & 88.5 & 11.5 & 0.0 & $6.57(0.03)$ \\
\hline \multicolumn{6}{|l|}{ Factor III } \\
\hline \multicolumn{6}{|l|}{ Obesity: } \\
\hline (a) routine exam & (3) & 76.1 & 23.7 & 0.2 & $6.11(0.04)$ \\
\hline (b) preconception care & (7) & 82.7 & 17.3 & 0.0 & $6.30(0.04)$ \\
\hline \multicolumn{6}{|l|}{ Exercise: } \\
\hline (a) routine exam & (4) & 67.0 & 31.4 & 1.6 & $5.87(0.05)$ \\
\hline (b) preconception care & $(10)$ & 68.3 & 31.0 & 0.7 & $5.94(0.05)$ \\
\hline \multicolumn{6}{|l|}{ General Nutrition: } \\
\hline (a) routine exam & $(6)$ & 51.2 & 46.7 & 2.1 & $5.51(0.06)$ \\
\hline (b) preconception care & $(8)$ & 82.2 & 17.5 & 0.4 & $6.28(0.04)$ \\
\hline \multicolumn{6}{|l|}{ Factor IV } \\
\hline \multicolumn{6}{|l|}{ Chronic diseases: } \\
\hline (a) routine exam & $(5)$ & 63.8 & 34.9 & 1.2 & $5.79(0.05)$ \\
\hline (b) preconception care & $(6)$ & 88.3 & 10.8 & 0.9 & $6.45(0.04)$ \\
\hline \multicolumn{6}{|c|}{ Family health history (inherited disorders): } \\
\hline (a) routine exam & $(8)$ & 51.5 & 43.9 & 4.6 & $5.38(0.06)$ \\
\hline (b) preconception care & (5) & 90.6 & 9.2 & 0.2 & $6.55(0.04)$ \\
\hline \multicolumn{6}{|c|}{ Over the counter and prescription drug use: } \\
\hline (a) routine exam & (9) & 42.0 & 51.4 & 6.5 & $5.08(0.06)$ \\
\hline (b) preconception care & (9) & 81.6 & 17.7 & 0.7 & $6.27(0.04)$ \\
\hline \multicolumn{6}{|l|}{ Environmental concerns: } \\
\hline (a) routine exam & (11) & 19.9 & 66.3 & 13.9 & $4.25(0.06)$ \\
\hline (b) preconception care & (11) & 56.1 & 39.1 & 4.8 & $5.47(0.06)$ \\
\hline
\end{tabular}

Table 5 Patient use of pre-pregnancy planning

Percent of physicians selecting a particular response to questionnaire items:

\begin{tabular}{|c|c|c|c|c|}
\hline \multirow{2}{*}{ How frequently do you have patients present for PCC? } & \multicolumn{2}{|c|}{ Never or rarely } & Sometimes & Frequently \\
\hline & \multicolumn{2}{|l|}{32.3} & 48.5 & 17.3 \\
\hline \multirow{2}{*}{$\begin{array}{l}\text { Women that I see usually do not plan their pregnancies } \\
\text { (on a } 5 \text { point scale) }\end{array}$} & \multicolumn{2}{|c|}{ Agree (1-2) } & Neutral (3) & Disagree (4-5) \\
\hline & \multicolumn{2}{|l|}{34.7} & 33.8 & 31.6 \\
\hline \multirow{2}{*}{$\begin{array}{l}\text { How many of your pregnant patients came in for } \\
\text { preconception care before they became pregnant? (OB) }\end{array}$} & None & Few & Some & Half or More \\
\hline & 3.3 & 45.8 & 38.3 & 12.6 \\
\hline \multirow{2}{*}{$\begin{array}{l}\text { How many of your pregnant patients initially made } \\
\text { contact with you once they were already pregnant? (OB) }\end{array}$} & All & Most & Many & Half or Fewer \\
\hline & 9.4 & 53.7 & 18.0 & 18.9 \\
\hline
\end{tabular}


frequently they perceived their patients to be utilizing PCC. Our findings document that most physicians think it is an important issue, that it has a positive effect on pregnancy outcomes, and that they are appropriately trained to provide it (Table 3). However, respondents reported that few of their patients seek PCC. Explanations for this lack of patients seeking PCC likely include the fact that almost $50 \%$ of pregnancies in the U.S. are unplanned [7], the lack of third party reimbursement for PCC visits, and patients' rather poor understanding of the potential benefits of PCC to the lifetime health of future offspring. We did find an association between the frequency with which physicians recommend PCC and the frequency with which patients present for PCC. It is also possible that some women receiving routine health care prior to a first pregnancy see an internist, family physician, or nurse practitioner for such care, and only seek the care of an obstetrician-gynecologist once they become pregnant. Thus, it is possible that our study of obstetrician-gynecologists does not reflect the full extent to which women may be seeking PCC. However, a recent study of primary care providers (not obstetrician-gynecologist) also found that few patients were receiving PCC [10].

Stumbling blocks to increasing utilization of PCC may include physicians' beliefs that time devoted to PCC is not reimbursed and that there is not enough time to provide PCC to all women of childbearing age. Regarding this latter point, opinions differ within the medical community as to who the target population for PCC should be. Three general target groups include women at high risk for poor birth outcome, women planning a pregnancy, and all women of childbearing age [11]. In this study, the vast majority of physicians said they frequently recommend PCC to diabetic and obese women planning a pregnancy, as well as to women generally who are planning a pregnancy (see Table 2). Far fewer obstetrician-gynecologists said they frequently recommend PCC to women described simply as sexually active (54\%) or to those using birth control (36\%). This suggests that our survey sample population views women who have consciously decided to plan a pregnancy as the most appropriate target group for PCC. Almost 9 in 10 (87\%) physicians defined PCC as specialized pre-pregnancy care focusing on issues not typically addressed during a routine exam, and the remaining $13 \%$ defined it as routine well-woman care occurring during the reproductive years, prior to a pregnancy. Those who defined it as routine were more likely to offer such counseling to women who were sexually active or using birth control than were those defining it as specialized care.

One aspect of PCC includes counseling women on topics that encourage a healthy pregnancy, although many such topics are clearly relevant to the general health of any woman. Physicians were asked to rate eleven topics in terms of their importance for counseling during PCC and routine care, such as exercise, nutrition, and over the counter drug use (see Table 4). All were considered very important topics for counseling in PCC, with cigarette smoking and folic acid supplementation rated the most highly important for PCC. For counseling in routine care, folic acid supplementation received the second lowest score of all, with under two-fifths (37\%) of physicians rating it as important. This points to the need for continued national attention to increase consumption and supplementation of folic acid.

Our study has limitations that should be acknowledged. The response rate was $60 \%$, and our findings are based on the responses of 579 non-subspecialist obstetricians and gynecologists. While our study may be subject to non-response bias, we believe that our findings are reliable. The typical response rate in these survey studies is approximately 35$60 \%$, and our response rate was at the high end of expected participation. In addition, the responses were derived from geographically diverse locations and from physicians from different practice types reflecting the influence of physician location and practice type. Whereas physicians who were more interested in the topic of the survey may have been more likely to respond, a subset of our subject pool was comprised of CARN members who respond to several questionnaires a year covering a wide variety of topics; it is unlikely that Preconception Care is a topic of greater interest to this group than to the group of randomly selected ACOG members. CARN members differed significantly from nonCARN subjects on only one non-demographic response, and the mean age and male to female ratio of respondents closely matched those of the larger group to whom the survey was sent and of ACOG Fellows and Junior Fellows as a whole, all of which suggests that response bias was minimized.

Recent studies have recommended offering PCC "opportunistically," incorporating information relevant to preconception care into routine well-woman visits $[10,12]$. Most topics relevant to preventing congenital birth defects are also important to the overall health of women, pointing out the compatibility of the two counseling contexts. The one exception to this in our questionnaire was folic acid supplementation, and it would need greater emphasis in routine care to take advantage of opportunistic counseling. We found that those physicians who defined PCC as "the same as routine well-woman care" differed from those defining it as "specialized" in several ways, including increased importance of counseling on folic acid supplementation during routine care. A recent study found that topics such as vitamin supplements and alcohol use were rarely discussed during routine care by a high proportion of obstetrician-gynecologists, and by an even higher proportion of family practitioners [13]. They also found that physician recommendations influenced women's decisions to take folic acid.

Obstetrician-gynecologists recognize the importance of preconception care and provide this care for their patients. 
However, lack of third party reimbursement, lack of time during office visits due to competing demands, and lack of consumer awareness pose barriers to effective implementation of PCC. The fact that almost half of all pregnancies in the U.S. are unplanned poses an even greater challenge. Continued efforts are needed to raise awareness of the importance of PCC by consumers, health care providers, third party carriers, and policy makers.

Acknowledgements This work was supported in part by a grant from the HRSA-MCHB and the CDC.

\section{References}

1. American Academy of Pediatrics, the American College of Obstetricians and Gynecologists. Guidelines for Perinatal Care. 5th Edition. 2002.

2. Ray JG, O'Brien TE, Chan WS. Preconception care and the risk of congenital anomalies in the offspring of women with diabetes mellitus: a meta-analysis. Q J Med 2001;94:435-44.

3. Centers for Disease Control and Prevention (CDC). Spina bifida and anencephaly before and after folic acid mandate-United States, 1995-1996 and 1999-2000. MMWR 2004;53:362-5.

4. de Jong-Van den Berg LT, Hernandez-Diaz S, Werler MM, Louik C, Mitchell AA. Trends and predictors of folic acid awareness and periconceptional use in pregnant women. Am J Obstet Gynecol 2005;192:121-8.

5. Holing EV, Beyer CS, Brown ZA, Connell FA. Why don't women with diabetes plan their pregnancies? Diabetes Care 1998;21:889 95.

6. NSDUH Report: substance use during pregnancy: 2002 and 2003 update. Available at http://www.oas.samhsa.gov/2k5/ pregnancy/pregnancy.pdf. Retrieved August 11, 2005.

7. Henshaw SK. Unintended pregnancy in the United States. Fam Plann Perspect 1998;30:24-9.

8. Curtis M, Abelman S, Schulkin J. Do we practice what we preach? A review of actual clinical practice with regards to preconception care guidelines. Maternal Child Health. In print (this issue).

9. Power ML, Holzman GB, Schulkin J. Obstetrician-gynecologists' views on the health risks of obesity. J Repro Med 2001;46:941-6.

10. Heyes T, Long S, Mathers N. Preconception care; practice and beliefs of primary care workers. Fam Pract 2004;21:22-7.

11. de Weerd S, Steegers EA.The past and present practices and continuing controversies of preconception care. Community Genet 2002;5:50-60.

12. Moos MK. Preconceptional health promotion: progress in changing a prevention paradigm. J Perinat Neonatal Nurs 2004;18:213.

13. March of Dimes Birth Defects Foundation. Folic Acid and the prevention of birth defects: a national survey of pre-pregnancy awareness and behavior of women of childbearing age, 1995-2001. White Plains, NY: March of Dimes Birth Defects Foundation, 2002. 